\title{
Anatomía de una confusión: error diagnóstico de patología paranoide en víctimas de mobbing
}

\author{
Anatomia de uma confusão: erro diagnóstico de patologia paranoide em vítimas de mobbing \\ Anatomy of a misunderstanding: wrong diagnosis of paranoid pathology in victims of mobbing
}

\author{
Ángel Martínez-Hernáez', Leticia Medeiros-Ferreira²
}

i Professor titular de Antropologia Médica da Universitat Rovira i Virgili, Tarragona, Espanha.

2 Médica-psiquiatra da CSMA Nou Barris, Barcelona, Espanha.

Recibido: 22/7/2009 - Aceptado: 18/8/2009

\section{Resumen}

Contexto: Diversas investigaciones subrayan el alto riesgo de error diagnóstico de trastorno delirante y trastorno paranoide de la personalidad entre víctimas de mobbing o acoso psicológico en el trabajo (APT). Objetivo: Analizar hasta qué punto los síntomas asociados con el mobbing son confundidos con criterios de dos nosologías del espectro paranoide (trastorno delirante y trastorno paranoide de la personalidad). Métodos: Se realiza una revisión bibliográfica desde 1990 hasta Junio de 2009 en PubMed y SciELO. Resultados: La identificación de síntomas del espectro paranoide en las víctimas de mobbing no resulta consistente con la literatura que, en cambio, indica una fuerte presencia de síntomas del espectro del estrés postraumático (hasta el 92\%), aunque no se cumpla el criterio A1 de esta nosología. Se apuntan algunas causas del error diagnóstico, tales como la tendencia a confundir hipervigilancia (criterio D4 del trastorno por estrés postraumático en el DSM-IV-TR) con ideación paranoide, la existencia de un perfil defensivo en las víctimas de APT y la falta de reconocimiento por parte de los clínicos del impacto estresante y traumatizante del mobbing. Conclusión: Se requieren investigaciones longitudinales y mixtas (cualitativos/cuantitativos) para establecer criterios robustos de diagnóstico diferencial entre las manifestaciones clínicas asociadas al mobbing y los síntomas paranoides.

Martínez-Hernáez A, Medeiros-Ferreira L / Rev Psiq Clín. 2010;37(4):167-74

Palabras clave: Mobbing, acoso psicológico en el trabajo, trastorno delirante, trastorno paranoide de la personalidad, trastorno por estrés postraumático.

\section{Resumo}

Contexto: Diversos estudos evidenciam o alto risco de erro diagnóstico de transtorno delirante e transtorno da personalidade paranoide entre as vítimas de mobbing ou assédio psicológico no trabalho (APT). Objetivo: Analisar a associação dos sintomas atribuídos ao mobbing com os critérios de duas nosologias do grupo paranoide (transtorno delirante e transtorno da personalidade paranoide). Métodos: Realiza-se uma revisão bibliográfica de 1990 a junho de 2009 em PubMed e SciELO. Resultados: A identificação de sintomas paranoides em vítimas de mobbing não é congruente com a literatura científica. Por outro lado, evidencia-se uma forte presença de sintomas vinculados ao estresse pós-traumático (até 92\%), ainda que não apresente o critério A1 dessa patologia. Algumas causas de erro diagnóstico seriam a tendência a confundir a hipervigilância (critério D4 do transtorno de estresse pós-traumático do DSM-IV-TR) com ideação paranoide, a existência de um perfil defensivo nas vítimas de APT e o desconhecimento do impacto estressante e traumatizante do mobbing pelos clínicos. Conclusão: São necessários estudos longitudinais e com metodologias mistas (qualitativas/quantitativas) para estabelecer critérios sólidos de diagnóstico diferencial entre as manifestações clínicas atribuídas ao mobbing e os sintomas paranoides.

Martínez-Hernáez A, Medeiros-Ferreira L / Rev Psiq Clín. 2010;37(4):167-74

Palavras-chave: Mobbing, assédio psicológico no trabalho, transtorno delirante, transtorno da personalidade paranoide, transtorno por estresse pós-traumático.

\begin{abstract}
Background: Several studies point out the high risk of misdiagnosing delusional disorder and paranoid personality disorder in victims of mobbing or workplace harassment (WPH). Objective: To analyze the extent to which the symptoms attributable to mobbing are misidentified with criteria for two paranoid spectrum nosologies (delusional disorder and paranoid personality disorder). Methods: Literature review of PubMed and SciELO from 1990 to June 2009 . Results: The identification of paranoid spectrum symptoms in victims of mobbing is not consistent with the literature, which, by contrast, shows a notable presence of symptoms in the post-traumatic stress spectrum (as much as $92 \%$ ), although they do not meet the A1 criterion for this nosology. Some of the causes of wrong diagnosis are noted, such as a tendency to confuse hypervigilance (D4 criterion for post-traumatic stress disorder in DSM-IV-TR) with paranoid ideation, the existence of a defensive profile in victims of WPH, and lack of recognition on the part of clinicians of the stressful and traumatizing impact of mobbing. Discussion: Longitudinal and mixed methodology (qualitative and quantitative) studies are necessary in order to establish robust differential diagnosis criteria that clearly distinguish the clinical manifestations attributable to workplace harassment from paranoid spectrum symptoms.
\end{abstract}

Martínez-Hernáez A, Medeiros-Ferreira L / Rev Psiq Clín. 2010;37(4):0-

Keywords: Mobbing, workplace harassment, delusional disorder, paranoid personality disorder, post-traumatic stress disorder. 


\section{Introducción}

La Señora $M$ es una funcionaria competente y con larga experiencia en la administración pública, pero hace dos años fue trasladada al sótano de su centro de trabajo. Desde entonces no le ha sido encomendada ninguna tarea ni puede tener acceso a un ordenador para realizar sus funciones laborales - por otro lado ya inexistentes - ni recibe ninguna explicación de la dirección o de sus compañeros de trabajo sobre las razones de esta situación. La ansiedad y el insom-

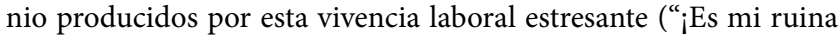
profesional!”) la ha llevado a visitar a un psicólogo y a ser tildada, como consecuencia del conocimiento de esta relación terapéutica por parte de sus compañeros de trabajo, de "loca", "enferma mental" y otro tipo de apelativos que han amplificado su proceso de denigración profesional y personal.

El Señor J es un albañil que debe soportar cada día la mofa de sus compañeros de trabajo, incluyendo al propietario de la empresa $y$ al jefe de obra, quienes parecen haber encontrado en su ligera tartamudez un motivo de escarnio y una excusa para la humillación continuada. "Bu bu bueenos días" suelen decirle por la mañanas protegidos en su pacto de agresión colectiva. J se siente dolido por esta experiencia, su sueño se ha perturbado, así como su atención y memoria. Se muestra hipervigilante e irascible, principalmente cuando las imágenes de denigración vuelven a su mente como un recuerdo insistente. Sabe que no puede actuar con violencia física ante los insultos y las vejaciones, pero también que las únicas alternativas son la baja laboral o el cambio de empresa.

La Señora D es biólogo y la más antigua de su laboratorio, sin embargo cada día se ve sometida a un cambio de rutinas y tareas, cada cual más compleja y difícil. Adicionalmente, sus compañeras hablan mal de ella a sus espaldas y nunca toman el desayuno con ella ni el almuerzo ni la invitan a las cenas de trabajo, a pesar de que cuando están solas solicitan su ayuda apelando a su proverbial competencia profesional. Después de una baja laboral por "trastorno adaptativo mixto con ansiedad y estado de ánimo depresivo" (DSM-IV-TR), la situación de acoso se amplificó. Ya no tiene que lidiar exclusivamente con los cambios de rutina, sino también con su estigma de "loca", a pesar de la inocuidad de su diagnóstico. En el último año, y como consecuencia de su traslado a otro centro de trabajo, su vida ha mejorado: "Me ha costado, pero ahora soy otra".

El Señor L es un profesor universitario con un buen currículum docente e investigador. Desde hace varios años, coincidiendo con la competencia por una posición estable, ha sido objeto de intromisiones y difamaciones sobre su vida privada, obstaculización de su carrera profesional, apropiación de su producción intelectual y calumnias malintencionadas que buscan su neutralización como potencial competidor, como que es un psicótico, un paranoico, un depresivo y una larga retahíla de diagnósticos improvisados. Es curioso que ni sus familiares ni sus amigos, entre los que se cuentan varios psiquiatras y psicólogos, estén de acuerdo con estos diagnósticos.

Todas estas situaciones pueden considerarse paradigmas de un fenómeno conocido en la literatura psiquiátrica, psicológica, médica, organizacional y legal de nuestro tiempo como mobbing, bullying adulto, workplace harassment, abuso emocional, acoso moral, acoso psicológico en el trabajo, psicoterror laboral o presión laboral tendenciosa, y que supone una clara vulneración de los derechos, la dignidad y la integridad física y psíquica de los trabajadores. En este texto vamos a utilizar los dos términos más comunes como sinónimos ( mobbing y acoso psicológico en el trabajo-APT) sin entrar a analizar esta diversidad terminológica ya profusamente debatida ${ }^{1-5}$.

El fenómeno del mobbing no es nuevo. Su definición primera por Leymann $^{1}$ se inspira en los estudios etológicos de Konrad Lorenz ${ }^{6}$ sobre el hostigamiento que puede practicar una manada o grupo de la misma o de diferentes especies a un único individuo de su misma especie o de otra. También guarda aires de familia con otros procesos sociales de denigración, violencia y hostilidad como el linchamiento, el bullying escolar, la violencia sexista, el racismo y la xenofobia, donde generalmente la violencia simbólica y psicológica precede a la potencial violencia física ${ }^{7}$ y donde la diferenciación antecede a la diferencia. No en vano, en todos estos fenómenos se produce una naturalización de la agresión como algo permisible entre un determinado grupo, así como un intento de deslegitimación de la víctima. Es la actualización de la lógica del chivo expiatorio que puso en evidencia René Girard8 en sus ensayos históricos y antropológicos.

Pero si el fenómeno del mobbing ya es antiguo, su tipificación legal es reciente. Concretamente se retrotrae al trabajo de Leymann como inspector laboral en Suecia en la década de los ochenta y a su regulación en este país en 19931,2,9. Desde entonces, diversos países (Francia, Holanda, Bélgica, España, entre otros) y estados (Québec, por ejemplo) han emprendido la adaptación de sus marcos jurídicos para paliar y penar este tipo de violencia, así como para proteger los derechos de los afectados ${ }^{10-12}$. Este proceso ha provocado debates sobre la naturaleza jurídico-penal de este fenómeno y sobre sus problemas de definición y discriminación ${ }^{13}$. Incluso en países como Suecia se ha procedido ya a evaluar el impacto de las políticas antimobbing, señalando sus éxitos y limitaciones". También ha dado pie a una abundante literatura en la psicología de las organizaciones y el management, por sus implicaciones en la caída de la productividad, la conflictividad en las empresas y las bajas laborales ${ }^{3,14}$.

Sin embargo, este desarrollo ha tenido poca trascendencia en el ámbito clínico, donde paradójicamente las demandas de atención de los síntomas asociados al mobbing cada vez son más frecuentes. Son escasas las investigaciones clínicas sobre APT, salvo algunas excepciones que posteriormente analizaremos, y este silencio no señala que exista un acuerdo o consenso con respecto a la atención de los afectados, sino discrepancias que se intentaban subrayar mediante las dos citas que abrían este artículo. Estas disensiones van más allá de la anécdota y permiten pensar en predisposiciones estructurales que pueden propiciar errores diagnósticos y malas prácticas.

En sus trabajos pioneros, Leymann ${ }^{1,2,15-17}$ observó un riesgo frecuente de error diagnóstico entre los afectados por mobbing que se ha convertido en una constatación recurrente entre los especialistas s-4,15-18. $^{\text {. }}$ Leymann ${ }^{1,2}$ y Piñuel ${ }^{3}$, entre otros, han aludido al error de atribución que se produce en el entorno clínico, concretamente la confusión entre la hipervigilancia reactiva al acoso y la paranoia; error que transforma la primera en la segunda por una falta de competencia clínica, por una incorrecta evaluación de los casos o por ambas cosas a la vez. El afectado se ve en estas situaciones doblemente victimizado y estigmatizado: por la empresa y por los servicios de salud, con la posibilidad de que se produzca un fenómeno devastador de exclusión y un alto riesgo de suicidio. Ahora bien, ¿A qué se debe este error de diagnóstico que, sin duda, requiere de una aclaración y análisis? Este artículo aborda esta cuestión a partir de una revisión sistemática de la literatura.

\section{Métodos}

Con el objetivo de explorar el diagnóstico diferencial entre patología paranoide y síntomas asociados al APT, se realizó una revisión sistemática (desde inicios de 1990 hasta junio de 2009) en PubMed a partir de categorías como mobbing o workplace harassment o bullying at work y delusional disorder, paranoid personality disorder, paranoia, diagnosis y posttraumatic stress disorder. Se establecieron los siguientes límites: adultos (+ 19 años, a fin de descartar el bullying escolar), humanos (para descartar los estudios etológicos en no-humanos) y artículos redactados en inglés, francés, portugués, italiano y español. Adicionalmente se realizó una revisión en SciELO y se procedió en forma de snowball (análisis acumulativo de red de citaciones) para llegar a fuentes no indexadas (libros, capítulos de libro, informes, etc.), entre las cuales se procedió por saturación cualitativa: hasta la obtención de redundancia.

\section{Resultados y discusión}

Con la metodología y los límites de búsqueda señalados se obtuvieron 207 referencias en PubMed para mobbing or workplace harassment or bullying at work, 28 en su relación con diagnosis, 1 con delusional disorder, 2 con paranoid personality disorder, 3 con paranoia y 12 con posttraumatic stress disorder. Asimismo se obtuvieron 14 referencias 
en SciELO, de las cuales se descartaron 2 por versar sobre especies animales. Mediante el sistema de snowball se obtuvieron 56 referencias destacadas. Los criterios para la selección e inclusión fueron el enfoque clínico, la pertinencia temática y su centralidad (número de citaciones). En total se revisaron 182 referencias y seleccionaron 65 . Ninguna de las referencias indexadas tenía como tema central el diagnóstico diferencial entre patología paranoide y sintomatología asociada al mobbing, aunque sí se tratase de forma periférica. A continuación se revisan y discuten los contenidos más relevantes.

El mobbing ha sido definido como la mayor amenaza actual para la salud de los trabajadores y se caracteriza como un tipo de violencia psicológica que es ejercida en el ámbito laboral de forma deliberada y continuada hacia un sujeto aislado por parte de otro u otros de su mismo entorno que pueden tener una relación jerárquica en relación al acosado, simétrica o subordinada, aunque esta última

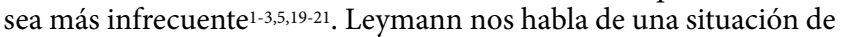
indefensión que se convierte en estructural y de un encadenamiento del acoso que como mínimo se prolonga a lo largo de 6 meses con acciones hostiles frecuentes (al menos 1 vez por semana) que en la mayoría de los casos son "silenciosas" o poco explícitas. En su LIPT (Leymann Inventory of Psychological Terrorization) distingue 45 tipos de acciones de hostigamiento. González de Rivera y RodríguezAbuín ${ }^{22-26}$ han realizado una ampliación y actualización de este cuestionario que puede visualizarse en la tabla 1: el LIPT-60, aunque sin su escala tipo Likert que oscila entre 0 y 4 . Otros cuestionarios y escalas conocidas son el Negative Acts Questionnaire (NAQ) ${ }^{27}$ y el Barómetro Cisneros ${ }^{19,28,29}$.

Si bien Leymann incluye la violencia física y situaciones de intimidación manifiestas, es consciente que la mayoría de las acciones se asocian con formas de violencia simbólica y psicológica sutiles que, tomadas de forma aislada, podrían parecer anodinas, pero cuya reiteración en el tiempo producen un efecto de estrés continuado y linchamiento moral. Los trabajos de Hirigoyen ${ }^{4}$, Piñuel ${ }^{3}$ y la mayoría de autores ${ }^{22-26}$ refuerzan esta idea. En realidad, el APT, como el insulto moral que tan bien ha analizado Cardoso de Oliveira ${ }^{7}$, suele mostrar una "apariencia de inmaterialidad" y, por tanto, tiende a ser invisibilizado como una agresión que merezca una reparación. Esta "apariencia de inmaterialidad" o invisibilidad ha sido analizada también en otros contextos, como en el bullying escolar ${ }^{30,31}$ y es uno de los subterfugios utilizados para el mantenimiento del APT, para sus intentos de justificación y para el error diagnóstico.

En el APT no todo es hostigamiento explícito o implícito. También éste se entremezcla con acciones de encubrimiento como el paternalismo, que mientras disimulan la agresión pasada actúan como mecanismo de mistificación del hostigamiento futuro. No por azar, algunas de los afectados relatan que los hostigadores acompañan o alternan el proceso de denigración con expresiones del tipo: "Eres como un hijo/a para mí" o "Con todo lo que yo he hecho por ti"; excusas que a la vez que introducen elementos de perversión en las relaciones laborales, pues se arrogan vínculos inexistentes, disimulan el mobbing de cara a terceros o al propio afectado, quien falto de la información necesaria como consecuencia de su aislamiento en las redes informales ( $y$ a veces también formales) de información de su institución actúa ante este tipo de situaciones con confusión e incluso autocrítica, adjudicándose una culpabilización que no le corresponde.

La mayoría de los expertos apuntan que la finalidad fundamental del mobbing es la salida de la organización por parte del afectado, aunque existen razones para matizar esta generalización. Se produce una confusión recurrente entre la agresión y su finalidad que está vinculada a la prueba legal en los tribunales de justicia y que debe ser subrayada. Como en la mayoría de conductas violentas, los fines pueden ser diversos. El mobbing puede responder a objetivos vicarios al despido, como la neutralización del afectado, la posibilidad de su control y dominación, la anulación de su empleabilidad futura, el boicot a su reputación para impedir que se enrole en otra institución competidora, la obstaculización de su carrera profesional y su traslado a otras dependencias. Siguiendo a Kleinman ${ }^{32-35}$, podemos decir que, como en el estigma, todo depende de lo que "esté en juego en un mundo moral local”. Por ejemplo, entre los funcionarios públicos, donde la dificultad objetiva del despido es bien conocida y a la vez las situaciones de APT son más frecuentes, suelen operar más las finalidades de denigración, neutralización, marginación, baja laboral o traslado del hostigado. De hecho, la mayor insistencia que se ha producido en el despido está asociada a que el campo de la empresa privada es, paradójicamente, donde la psicología de las organizaciones ha tenido un mayor desarrollo.

El mobbing tiene su origen en el entorno laboral, pero muestra una prolongación más allá de él, como es la arena profesional y asociativa, por no hablar aquí de la familiar e interpersonal ${ }^{1-4}$. En la medida que el objetivo del APT es la neutralización y denigración del afectado y que los colectivos profesionales están conformados por redes sociales, es lógico pensar que puedan verse envueltos en este proceso, redundando, o no, en la estigmatización y la exclusión. Lógicamente esto supera la competencia de la organización, pero no por ello debe obviarse a la hora de hacer un análisis holístico. Prueba de ello es que muchos hostigados no vuelven a tener un empleo una vez que lo han perdido por despido o por una baja laboral ${ }^{2}$. Esta constatación puede asociarse en los casos más graves al deterioro físico y/o psicológico, pero también al hundimiento de su reputación en el campo profesional.

El mobbing es un fenómeno que presenta diferentes fases e intensidades. Habitualmente se habla de diversas etapas que dibujan una dinámica in crescendo, aunque esta tipología debe entenderse como una orientación general, pues hay casos que pueden guardar características diferenciadas dependiendo de la toma de conciencia del fenómeno por parte del afectado (la verbalización del mobbing modifica el mobbing), del apoyo de las redes sociales y de la actitud de la organización, entre otros factores ${ }^{1-4}$. En la tabla 2 se resumen dos de las tipologías más utilizadas ${ }^{1,3}$ junto con el estado clínico y los síntomas que García de la Rivera y López García-Silva ${ }^{36}$ han asociado con las diferentes fases.

La epidemiología del mobbing presenta resultados contradictorios dependiendo de las escalas utilizadas para su medición y de los sectores laborales analizados ${ }^{37-39}$. En una revisión de estudios realizados en Europa, Zapf et al. 40 establecen que en su modalidad más severa afectaría entre el $1 \%$ y el $4 \%$ de los trabajadores y entre el $8 \%$ y el $10 \%$ en su tipología más tenue. Según Di Martino ${ }^{41}$, se podría hablar también de una diferencia de prevalencia según el carácter episódico o crónico del fenómeno: $10 \%$ y $5 \%$, respectivamente. En España, los diferentes informes Cisneros permiten estimar que el problema afecta al 15\% de los trabajadores en activo y, en su versión $\mathrm{V}$, focalizada en los funcionarios públicos, apunta una prevalencia de hasta el 25\%19,28,29. Los sectores profesionales más afectados son la administración pública, la educación y la sanidad.

Si bien el mobbing no es una enfermedad, sino un comportamiento lesivo, sus efectos en la salud de los trabajadores son evidentes y en algunos casos han dado pie a confusiones diagnósticas que son objeto de esta revisión. Los síntomas y malestares más frecuentes suelen estar vinculados al estrés manifestado y a menudo articulado en el ciclo oscilatorio alerta-depresión-defensividad ${ }^{1-4,42-49}$, como el dolor de espalda y articular, los diversos síntomas psicosomáticos, el trastorno del sueño, la falta de concentración y atención, las alteraciones de la memoria, la ansiedad y la depresión reactivas, la fatiga crónica, la hipervigilancia reactiva, la irritabilidad y el aislamiento social, entre otros muchos.

Estos síntomas son altamente inespecíficos y pueden formar parte de cualquier cuadro psicológico/psiquiátrico sin por ello suponer su categorización como trastorno. En su conjunto, y si la intensidad de la sintomatología altera el funcionamiento cotidiano del afectado, se pueden identificar como manifestaciones del llamado "trastorno de estrés por coacción continuada" (prolonged duress stress disorder - PDSD) ${ }^{50}$, el cual adopta los mismos síntomas que el trastorno por estrés postraumático ${ }^{50-54}$, aunque no se cumplan todos los criterios diagnósticos de esta última nosología (especialmente no se cumple el criterio A1 del DSM-IV-TR). Por otro lado, algunas estimaciones afirman que el mobbing aparece estrechamente relacionado con otras enfermedades (dolencias coronarias, cáncer, hipertensión, trastornos digestivos, etc.), con comportamientos de riesgo (adicciones, accidentalidad viaria y laboral) y con el suicidio ${ }^{1-4,55-58}$. 
Tabla 1. LIPT-60 (Leymann Inventory of Psychological Terrorization) modificado por González de Rivera y Rodríguez-Abuín22-26

1. Sus superiores no le dejan expresarse o decir lo que tiene que decir.

2. Le interrumpen cuando habla.

3. Sus compañeros le ponen pegas para expresarse o no le dejan hablar.

4. Le gritan o le regañan en voz alta.

5. Critican su trabajo.

6. Critican su vida privada.

7. Recibe llamadas telefónicas amenazantes, insultantes 0 acusadoras.

8. Se le amenaza verbalmente.

9. Recibe escritos y notas amenazadoras.

10. No le miran, o le miran con desprecio o gestos de rechazo.

11. Ignoran su presencia, no responden a sus preguntas.

12. La gente ha dejado o está dejando de dirigirse o de hablar con usted.

13. No consigue hablar con nadie, todos le evitan.

14. Le asignan un lugar de trabajo que le mantiene aislado del resto de sus compañeros.

15. Prohíben a sus compañeros que hablen con usted.

16. En general, se le ignora y se le trata como si fuera invisible.

17. Le calumnian y murmuran a sus espaldas.

18. Hacen circular rumores falsos o infundados sobre usted.

19. Le ponen en ridículo, se burlan de usted.

20. Le tratan como si fuera un enfermo mental o lo dan a entender.

21. Intentan obligarle a que se haga un examen psiquiátrico o una evaluación psicológica.

22. Se burlan de alguna deformidad o defecto físico que pueda tener.

23. Imitan su forma de andar, su voz, sus gestos para ponerle en ridículo.

24. Atacan o se burlan de sus convicciones políticas o de sus creencias religiosas.

25. Ridiculizan o se burlan de su vida privada.

26. Se burlan de su nacionalidad, procedencia o lugar de origen.

27. Le asignan un trabajo humillante.

28. Se evalúa su trabajo de manera parcial, injusta y malintencionada.

29. Sus decisiones son siempre cuestionadas o contrariadas.

30. Le dirigen insultos o comentarios obscenos o degradantes.

31. Sufre acercamientos, insinuaciones o gestos sexuales no deseados.

32. No se le asignan nuevas tareas, no tiene nada que hacer.
33. Le cortan sus iniciativas, no le permiten desarrollar sus ideas.

34. Le obligan a hacer tareas absurdas 0 inútiles.

35. Le asignan tareas muy por debajo de su competencia.

36. Le sobrecargan sin cesar con tareas nuevas y diferentes.

37. Le obligan a realizar tareas humillantes.

38. Le asignan tareas muy difíciles o muy por encima de su preparación, en las que es muy probable que fracase.

39. Le obligan a realizar trabajos nocivos o peligrosos.

40. Le amenazan con violencia física.

41. Recibe ataques físicos leves, como advertencia.

42. Le atacan físicamente sin ninguna consideración.

43. Le ocasionan a propósito gastos para perjudicarle.

44. Le ocasionan daños en su domicilio o en su puesto de trabajo.

45. Recibe agresiones sexuales físicas directas.

46. Ocasionan daños en sus pertenencias o en su vehículo.

47. Manipulan sus herramientas (por ejemplo, borran archivos

de su ordenador).

48. Le sustraen algunas de sus pertenencias, documentos o herramientas de trabajo.

49. Se someten informes confidenciales y negativos sobre usted, sin notificarle ni darle oportunidad de defenderse.

50. Las personas que le apoyan reciben amenazas, o presiones

para que se aparten de usted.

51. Devuelven, abren o interceptan su correspondencia.

52. No le pasan las llamadas, o dicen que no está.

53. Pierden u olvidan sus encargos, o los encargos para usted.

54. Callan o minimizan sus esfuerzos, logros, aciertos y méritos.

55. Ocultan sus habilidades y competencias especiales.

56. Exageran sus fallos y errores.

57. Informan mal sobre su permanencia y dedicación.

58. Controlan de manera muy estricta su horario.

59. Cuando solicita un permiso o actividad a la que tiene derecho se lo niegan o le ponen pegas y dificultades.

60. Se le provoca para obligarle a reaccionar emocionalmente.

Tabla 2. Fases del mobbing según diferentes autores

\begin{tabular}{|c|c|c|}
\hline Fases del mobbing según Leymann1 & Fases del mobbing según Piñuel ${ }^{3}$ & La clínica según González-de-Rivera y López García-Silva36,23 \\
\hline $\begin{array}{l}\text { 1. Incidentes críticos. Conflicto inicial que provoca una } \\
\text { escalada. No constituye propiamente mobbing y suele durar } \\
\text { poco tiempo }\end{array}$ & 1. Incidentes críticos. (Igual que Leymann) & $\begin{array}{l}\text { Estadio clínico: Autoafirmación } \\
\text { Síntomas: Inquietud, agresividad moderada, caben } \\
\text { alteraciones del sueño }\end{array}$ \\
\hline $\begin{array}{l}\text { 2. Mobbing y estigmatización. Se desarrollan } \\
\text { comportamientos repetidos que en un contexto distinto no } \\
\text { revestirían mayor importancia. Sin embargo, su frecuencia y } \\
\text { periodicidad con propósitos hostiles estigmatizan a la víctima }\end{array}$ & $\begin{array}{l}\text { 2. Fase de acoso y estigmatización. (Igual que Leymann). } \\
\text { Utilización de las alteraciones de la atención, memoria, } \\
\text { hipervigilancia, etc., producidas por el mobbing como } \\
\text { justificaciones del mismo }\end{array}$ & $\begin{array}{l}\text { Estadio clínico: Desconcierto. } \\
\text { Síntomas: Dudas sobre su versión, pérdida de } \\
\text { autoconfianza, frustración. Empeoramiento de los } \\
\text { síntomas de la fase anterior }\end{array}$ \\
\hline $\begin{array}{l}\text { 3. Intervención de la dirección de la empresa. } \\
\text { Formalización del caso y también de la estigmatización de la } \\
\text { víctima. Transgresión de derechos básicos del trabajador }\end{array}$ & $\begin{array}{l}\text { 3. Intervención de la dirección (responsables } \\
\text { jerárquicos). Error de atribución que lleva a que compañeros, } \\
\text { jefes y directivos tiendan a elaborar explicaciones basadas en } \\
\text { las características individuales de la víctima, en lugar de en los } \\
\text { factores del entorno }\end{array}$ & $\begin{array}{l}\text { Estadio clínico: Depresivo } \\
\text { Síntomas: Cansancio emocional, evitación y aislamiento, } \\
\text { inadecuación y culpa. Empeoramiento de los síntomas } \\
\text { de las fases anteriores }\end{array}$ \\
\hline \multirow[t]{2}{*}{$\begin{array}{l}\text { 4. Expulsión. Salida anticipada del mundo laboral. Aparición } \\
\text { de síntomas que llevan a la víctima a la búsqueda de ayuda } \\
\text { profesional. Errores diagnósticos, principalmente paranoia, } \\
\text { depresión-maniaca y trastorno de la personalidad }\end{array}$} & $\begin{array}{l}\text { 4. Fase de solicitud de ayuda especializada externa y } \\
\text { diagnóstico incorrecto. Errores diagnósticos comunes: } \\
\text { personalidad paranoide, depresión-maníaca o ciclotimia, } \\
\text { desajuste de la personalidad, etc. } \\
\text { “Una vez que la organización tiene conocimiento de que } \\
\text { la víctima recibe algún tipo de tratamiento (información } \\
\text { que a veces es inventada o fabulada, o es dada a } \\
\text { conocer por el acosador), ello sirve para reforzar la } \\
\text { estigmatización }\end{array}$ & $\begin{array}{l}\text { Estadio clínico: Traumático } \\
\text { Síntomas: Estrés-ansiedad, intrusiones obsesivas, } \\
\text { conflictos múltiples (también familiares) }\end{array}$ \\
\hline & $\begin{array}{l}\text { 5. Salida de la organización. Dimisión de la víctima, } \\
\text { traslado, excedencia, baja laboral. Difícil retorno al } \\
\text { empleo. Empleabilidad dinamitada. Potencial suicidio }\end{array}$ & $\begin{array}{l}\text { Estadio clínico: Estabilización crónica } \\
\text { Síntomas: Incapacidad laboral, presión focalizada del } \\
\text { pensamiento, hostilidad-depresión, aumento de bajas } \\
\text { laborales (empeora cerca de la vuelta al trabajo), temor } \\
\text { a lugar y recuerdos }\end{array}$ \\
\hline
\end{tabular}

Parece obvio, pero quizá necesario de subrayar aquí, que no todos los afectados enferman ni tienen atributos de vulnerabilidad diferenciales, ya sea en términos físicos o psicológicos. El consenso de los expertos es que se trata de una conducta lesiva que puede afectar a cualquier trabajador y que, en la medida en que está orientada a minarlo psicológicamente, es lógico que acabe teniendo algún efecto, 
sobre todo si se prolonga en el tiempo. Podríamos establecer una gradación que se iniciaría en la normalidad, continuaría con la aparición de síntomas con entidad subclínica, cuya atención podría ubicarse (si seguimos la lógica del DSM-IV-TR) en "otros problemas que pueden ser objeto de atención clínica", concretamente "problema laboral" (Z56.7), y finalmente desembocaríamos en la aparición de trastornos y enfermedades francos que se asocian ya con la baja por enfermedad, entre las cuales estaría el trastorno adaptativo, la depresión mayor y el trastorno por estrés postraumático (sin el criterio A1).

No debe descartarse que el APT pueda producir o desencadenar trastornos mentales graves, entre ellos el trastorno delirante (la anteriormente denominada paranoia), el trastorno paranoide de la personalidad y el trastorno bipolar (antes psicosis maniaco-depresiva). Éstos son los diagnósticos erróneos habituales según Leymann. También el APT puede conformarse como tema central de una ideación delirante, por lo que estaríamos ante un caso de falso mobbing59. Incluso puede coexistir la existencia de un trastorno mental o de la personalidad y APT debido a la consustancial estigmatización de los problemas de salud mental. Estas posibilidades no son puestas en duda en la literatura sobre el mobbing ni deben ser descartadas. No obstante, hay evidencia robusta de que estos fenómenos son poco prevalentes y de que generalmente el uso de nosologías como trastorno delirante y trastorno paranoide de la personalidad responde a un error clínico de atribución que redunda en el sufrimiento de los afectados. Veámoslo con mayor extensión.

1) El trastorno delirante de tipo paranoide - que es el mayor objeto de error diagnóstico - muestra una incidencia muy baja en la población general (menos del 0,03\%) según la APA (DSM-IVTR) ${ }^{60}$, mientras que las estimaciones más bajas de APT indican una prevalencia de este fenómeno entre el $1 \%$ y el $4 \%$ de la población trabajadora en activo, así como de tasas que pueden acercarse al $25 \%$ en los sectores laborales más vulnerables. Es por ello que la asociación generalizada entre sintomatología asociada al mobbing y trastorno delirante no resulta consistente. Por otro lado, la víctima de mobbing no sufre una ideación persecutoria, sino que es objeto de violencia y acoso. Aquí está el error de atribución mayor que tiene que ver con la confusión clínica entre paranoia e hipervigilancia congruente y reactiva ante APT por aumento de la activación (arousal) (criterio D4 del trastorno por estrés postraumático en el DSM-IV-TR). La paranoia supone una ideación delirante que suele ser estructural a la personalidad, está basada en una alteración del contenido del pensamiento y puede reproducirse en otros contextos y esferas. $\mathrm{La}$ reacción de alerta ante el acoso constituye una respuesta congruente con el contexto, oscila con síntomas depresivos y conduce finalmente a una sintomatología propia del estrés continuado o del estrés postraumático. A veces también de depresión mayor o trastornos del espectro ansioso, si reúne criterios diagnósticos suficientes para una u otra entidad. Como es obvio, y teniendo en cuenta el modelo clínico de fases de la tabla 2 y las investigaciones realizadas, sería muy improbable que el mismo paciente en su curso evolutivo transitase del trastorno delirante a la distimia/depresión y posteriormente al trastorno por estrés postraumático ${ }^{50-52,58}$. Resulta más factible pensar en un error diagnóstico que transforma la hipervigilancia por aumento de la activación (arousal) ("Me denigran en el trabajo y no puedo sacármelo de la cabeza, ni dormir") en un síntoma paranoide ("Cree que le denigran en el trabajo").

2) Una segunda nosología frecuentemente confundida con las manifestaciones clínicas del mobbing, el trastorno paranoide de la personalidad, muestra una prevalencia mayor que el delirante en la población general $(0,5 \%-2,5 \%)$ según la APA (DSM-IV-TR) ${ }^{60}$. La inconsistencia aquí - también aplicable al punto 1 - es que no se corresponde con los perfiles psicológicos y psicopatológicos más habituales entre los afectados y/o denunciantes de APT. Diversas investigaciones apuntan que no existe un perfil específico entre las víctimas, pues las razones del mobbing son exógenas al individuo afectado o en todo caso relacionadas con factores positivos, como su éxito profesional y su eficacia laboral, que han constituido el objetivo a neutralizar por parte del acosador o acosadores, quienes, en todo caso, sería o serían los sujetos susceptibles de recibir atención psicológica/psiquiátrica1-5. Otros estudios ${ }^{61,62}$ indican que pueden diferenciarse dos grupos: un primer grupo (en torno al 70\%) que muestra características de normalidad y un segundo grupo con variedad de perfiles, fundamentalmente del espectro neurótico. Hay investigaciones ${ }^{62-67}$ que han administrado el MMPI-2 (Minnesota Multiphasic Personality Inventory) entre afectados y muestran perfiles de trastorno de la personalidad más altos en víctimas que entre la población general, principalmente en lo que respecta al espectro neurótico y la somatización, así como altos niveles de ansiedad social en las relaciones interpersonales, entre otros rasgos, pero no suficientemente del perfil paranoide para establecer una asociación generalizada. Por ejemplo, en una investigación reciente ${ }^{65}$ González Trijueque y Delgado Marina compararon una muestra de 50 víctimas de APT con otra de trabajadores activos que actuaron como control. En ambos casos administraron el MMPI-2 y sólo diagnosticaron un caso de trastorno paranoide de la personalidad entre las víctimas frente a 9 con el perfil obsesivo-compulsivo, 4 con el perfil dependiente, 2 con el fóbico-evitativo y 2 con el histriónico en el mismo grupo. En síntesis, de los resultados de las investigaciones se colige que la asociación generalizada entre mobbing y trastorno paranoide de la personalidad no resulta consistente.

3) Rodríguez-Abuin y González de Rivera ${ }^{68}$ ofrecen una clave para entender el error de atribución que se produce en los entornos clínicos. Según estos autores, una parte considerable de los afectados por mobbing presentan ante el clínico rasgos que podrían ubicarse en el espectro "hostil-paranoide", mientras que otros muestran un perfil "depresivo-ansioso". Es preciso subrayar que los autores se refieren a rasgos no necesariamente patológicos. Siguiendo a estos autores, la cuestión es saber si el paranoidismo es reactivo a la experiencia traumática (paranógena) o estructural al paciente. En el primer caso, la existencia de paranoidismo no significa que el paciente sufra de un trastorno paranoide (trastorno delirante o trastorno paranoide de la personalidad), ya que su hipervigilancia está más contenida, es transitoria y reactiva, existe una necesidad de cercanía emocional y se apacigua ante el apoyo y comprensión de su historia; en palabras de los autores: "Tener síntomas o condiciones paranoides no implica ser paranoide o padecer un trastorno paranoide estructural" 68 . En el segundo caso (el estructural), sí que estamos ante una entidad clínica con hipervigilancia generalizada, tendente a la cronicidad, que no muestra necesidad de cercanía emocional y que es generalmente invariable al apoyo y la comprensión. Según estos autores, la aplicación del Test de Rorschach permite discriminar entre estos dos cuadros.

Probablemente sería útil acuñar otro concepto para definir el paranoidismo reactivo al acoso, pues presta a confusión con la paranoia estructural, fundamentalmente en el mundo profano a la psicopatología. No obstante, la distinción en sí misma es apropiada porque diferencia estados que no deberían confundirse. En realidad, podríamos hablar de un continuum asociado con el nivel de paranoidización: ausencia de manifestaciones clínicas $\rightarrow$ hipervigilancia congruente y reactiva al mobbing (por el aumento del arousal) $\rightarrow$ paranoia estructural. Las dos primeras son frecuentes entre víctimas de APT. La tercera es residual. De hecho, en un ambiente de hostilidad, lo "anormal" es no presentar síntomas/malestares reactivos a las experiencias vividas.

El trastorno paranoide estructural es susceptible al entorno pero no dependiente de él, por eso no se modifica cuando el contexto es diferente. La hipervigilancia reactiva al mobbing en cambio sí. La toxicidad del ambiente laboral se hace palpable cuando el sujeto tiene disponibilidad de cambiar de empresa u organización, aunque sea sólo por un cierto tiempo (excedencias, permisos, etc.), y tiende a olvidar la vivencia traumática, a eliminar su hipervigilancia y hostilidad, así como a arrinconar los síntomas de tipo depresivo y ansioso. Siempre, claro está, que su estado no haya alcanzado en la fase traumática de la tabla 2.

4) La víctima de mobbing se encuentra atrapada en el círculo vicioso de enfrentarse al acoso o aceptarlo. Esto explica la existencia de los dos grandes tipos "hostil-defensivo" y "depresivo-ansioso" que se observan en la práctica clínica y que, aunque no se indique con la suficiente rotundidad, generan oscilaciones en el mismo sujeto 
en diferentes momentos vitales como consecuencia de la naturaleza reactiva de estos estados ${ }^{61}$. La indefensión es uno de los polos, la defensividad el otro. Entre ellos se dilucida la partida. En sus extremos se juega también el riesgo de transformar la manifestación en un trastorno mental franco. Pero el riesgo que las investigaciones hasta ahora nos presentan en el mobbing no es la paranoia, sino la depresión, la ansiedad o el trastorno por estrés postraumático ${ }^{1-4,50-52}$, que no por azar son entidades clínicas más prevalentes. Piénsese que la concordancia entre APT y síntomas de trastorno por estrés postraumático puede alcanzar el $92 \%$; y lo que es más revelador: existe una asociación consistente entre la intensidad del acoso y el número e intensidad de los síntomas ${ }^{52}$. La reacción defensiva hacia el mobbing es en el fondo la compensación que permite al afectado mantener un funcionamiento "normalizado" (como era el previo a la situación de acoso) y no aceptar la indefensión. Es por ello que su diagnóstico erróneo produce un efecto extremadamente negativo que ancla a la víctima en una redundada indefensión, destruyendo su "sentido de coherencia" 69 e incrementando el riesgo de patología grave (depresión mayor, trastorno por estrés postraumático) y de suicidio ${ }^{1-4}$.

Por otro lado, el mobbing es un proceso largo y muchas veces discontinuo. Por ello es frecuente el error clínico de asociar la emergencia de sintomatología con factores ajenos al APT, así como el sesgo de interpretar los cambios en la autopercepción de la reputación profesional del hostigado como si fuesen fruto de sus conductas desafortunadas. Estos dos errores de apreciación clínica tienden a amplificar el problema del APT por redundar en el estigma y culpabilizar a la víctima.

5) Otro elemento a tener en cuenta es la presentación del afectado en el entorno clínico y su estado de confusión, que puede conllevar la verbalización de que está siendo agredido y denigrado en el entorno laboral pero sin mostrar elementos de "materialidad" o pruebas que ofrezcan consistencia a esta idea. Esta situación puede favorecer el error de juicio clínico de que se trata de ideas sobrevaloradas o incluso delirantes. Adicionalmente, en muchas ocasiones se produce una incomprensión en el propio ámbito familiar sobre la vivencia del mobbing ${ }^{1-4}$, principalmente porque antes de la fase 1 las relaciones personales que han generado el conflicto podían ser neutras o incluso buenas. La pareja y los familiares pueden mostrarse poco empáticos con la experiencia de la víctima y sus efectos derivados: irritabilidad, perturbación del sueño, hipervigilancia, problemas de concentración y atención, rememoración constante de los eventos traumáticos etc. $y$, por ignorancia o incredulidad, pueden pensar que el afectado/a es el causante de la situación o que todo es fruto de una alteración de su percepción. Como indica Piñuel ${ }^{3}$, es frecuente en estos casos que el error de atribución se instale accidentalmente también en la pareja, familia y amigos con la extendida frase: “¿Estás paranoico!". En estas situaciones que no son infrecuentes, la posición más sencilla para el clínico es refrendar esta afirmación sin realizar una evaluación en profundidad, por ejemplo administrando escalas y tests proyectivos o haciendo una exploración clínica y contextual exhaustiva. No obstante, la mayoría de afectados mostrarán características contradictorias con el espectro paranoide, como la empatía, la duda, la aceptación de la crítica a sus supuestas ideas delirantes o la autoculpabilización. Por otro lado, en los casos en que la pareja confirma el APT puede producirse el error de atribución clínica de folie à deux. Como relata S, marido de B que estaba siendo acosada en su centro de trabajo: "Fuimos al médico a explicar lo que le ocurría a mi mujer y nos dijo que teníamos manía persecutoria".

6) Las manifestaciones del mobbing se instalan en la polaridad depresivo-ansiosa y hostil-defensiva. Los riesgos de error diagnóstico que apuntaba tempranamente Leymann indican una lectura exacerbada de estas manifestaciones: trastorno bipolar o ciclotimia cuando se percibe clínicamente la oscilación entre los dos perfiles y trastorno delirante o trastorno paranoide de la personalidad en el espectro hostil-defensivo. Probablemente necesitamos un marco conceptual más elaborado de los denominados trastornos adaptativos que permita incluir la presentación de "hipervigilancia congruente y reactiva por APT o por otro tipo de situaciones de violencia" como una manifestación prevalente que, a su vez, cicla con un perfil depresivo-ansioso, también reactivo, y que se orienta hacia el trastorno por estrés postraumático.

7) Cabe también resaltar que los errores, las comorbilidades y la estabilidad en el tiempo de un diagnóstico dependen en gran medida de la forma como están estructurados los criterios diagnósticos. Dado que las enfermedades psiquiátricas no tienen límites precisos y en su mayor parte son de etiología desconocida, las nosologías creadas presentan limitaciones para definir dónde empieza y termina un cuadro nosológico. Esto facilita que un mismo sujeto pueda ser diagnosticado de padecer enfermedades diferentes, según el momento de la valoración del cuadro clínico. La forma de disminuir el porcentaje de errores es considerar que el diagnóstico no puede limitarse a una valoración transversal, sino que debe ser susceptible de modificarse según el clínico vaya componiendo el puzzle del diagnóstico psiquiátrico.

8) Generalmente la formación en APT entre los clínicos es muy deficiente, cuando no nula. A modo de ejemplo, podemos decir que en una investigación en curso que estamos realizando entre los profesionales en salud y salud mental de Barcelona (España), la mayoría de los sujetos entrevistados no habían leído nada sobre mobbing, ni en las revistas profesionales ni en los libros de divulgación, ni habían tenido acceso siquiera a información sobre su sintomatología asociada. Generalmente se vinculaba con obstáculos en el desarrollo del trabajo, pero no con los aspectos denigrantes asociados con los ataques a la dignidad personal, aunque estos últimos son los más frecuentes $(100 \%)^{52}$. En muchos casos no se mostraba el menor interés por acceder a información a este respecto, que se identificaba con una "nueva" o "falsa" patología y no con una conducta lesiva, cuando no se banalizaba como una realidad sobredimensionada por los expertos y por las víctimas. En algunas situaciones se trataba como un fenómeno ubicuo que todo el mundo padece o puede padecer, pero que no por ello genera síntomas, y que en todo caso éstos se deben a una vulnerabilidad individual del afectado o a características de su personalidad de base. Tampoco se establecía una identificación entre la vivencia del mobbing y el trastorno por estrés postraumático, pues se entendía que mientras el segundo deviene de acontecimientos claramente traumáticos, el primero no. De esta forma, las amenazas asociadas con el APT (pérdida del trabajo, ruina de la carrera profesional, pérdida del sustento económico, denigración y desfiguración de la persona en la esfera pública, atribución de una enfermedad mental en el entorno laboral, etc.) no eran valoradas como merecedoras de atención como factores "realmente" estresantes y traumatizantes. La inmensa mayoría parecía desconocer las investigaciones que apuntan que el efecto del estrés continuado tiene mayores implicaciones para la salud física y psicológica que las vivencias traumáticas de etiología impersonal que, por otro lado, suelen despertar una mayor solidaridad y empatía sociales, probablemente también entre los profesionales.

9) Otro factor a destacar es la presión que determinados entornos clínicos altamente burocratizados realizan con respecto a la "urgencia" de establecer un diagnóstico y que facilitan la atribución de nosologías más consensuadas que categorías difusas como la de trastorno adaptativo o por estrés postraumático ante condiciones de mobbing. En estas situaciones, la urgencia de obtener un diagnóstico, junto a actitudes dogmáticas de escuela y de banalización del mobbing, puede obliterar la posibilidad de una exploración no prejuiciosa. Como consecuencia de un diagnóstico apresurado y equivocado se desarrolla un plan terapéutico basado en un error que, en vez de dotar al paciente de estrategias de afrontamiento adecuadas ante APT, agravan su condición psicopatológica al interpretar clínicamente sus quejas como delirios.

10) En última instancia queremos citar un sesgo que tiene que ver con el entorno clínico. A diferencia de la psicología de las organizaciones y de las ciencias sociales aplicadas, más orientadas a analizar conflictos interpersonales en las instituciones que patologías, las disciplinas clínicas adoptan una tendencia inversa. De ahí se deriva una dificultad estructural para contextualizar los comportamientos individuales y los padecimientos. Es sabido que los factores sociales, culturales y psicosociales no son analizados in extenso en estos ámbi- 
tos, a pesar de que el DSM-IV-TR y otros manuales al uso indiquen la necesidad de un conocimiento del contexto para discernir, por ejemplo, una idea congruente con una ideación delirante e0-72 $^{70}$. De ello deviene una atomización e individualización del fenómeno del APT que se expresa muy bien en su transformación en una especie de patología difusa, incongruente y por tanto más fácilmente confundible con la paranoia. Al obliterar el clínico la capacidad interpretativa y el conocimiento del contexto social, cobra sentido la afirmación de Leymann ${ }^{1}$ que abría este texto: "Muy frecuentemente la víctima es diagnosticada incorrectamente, ya sea porque el profesional no se cree su historia personal o porque no se molesta en analizar la dinámica social desencadenante".

\section{Conclusiones}

En esta revisión se han apuntado algunas hipótesis exploratorias que requieren de ulteriores investigaciones. Las evidencias apuntan a la inconsistencia de la asociación generalizada entre los síntomas asociados al mobbing y el espectro paranoide como consecuencia de la confusión clínica entre la hipervigilancia reactiva y la paranoia estructural. También hay evidencia de que es posible distinguir ambos cuadros con técnicas proyectivas como el test de Rorschach y la exploración clínica en profundidad. Los afectados por mobbing tienden a ciclar entre las posiciones "depresivo-ansiosa" y "hostildefensiva”. Sus manifestaciones pueden ser subclínicas o clínicas. En estos últimos casos es relevante el diagnóstico diferencial entre el espectro paranoide y la hipervigilancia reactiva, también entre las víctimas de otras formas de violencia continuada. Como en otras situaciones clínicas ${ }^{73,74}$, son necesarias investigaciones mixtas (cuantitativas/cualitativas) y longitudinales para establecer criterios robustos de diagnóstico diferencial. También de comparaciones longitudinales entre víctimas de otras formas de violencia continuada y afectados por mobbing, así como investigaciones sobre posibles sesgos diagnósticos en ambos casos.

\section{Agradecimientos}

Los autores agradecen al editor sus comentarios y cortesía. También a los revisores anónimos por sus valiosas aportaciones, especialmente su lúcida síntesis del tema de este artículo: "confundir quejas con delirios".

\section{Referencias}

1. Leymann $\mathrm{H}$. The content and development of mobbing at work. Eur J Work Org Psych. 1996;5(2):165-84.

2. Leymann H. Mobbing and psychological terror at workplaces. Violence Vict. 1990 Summer;5(2):119-26.

3. Piñuel I. Mobbing. Santander: Sal Editores; 2001.

4. Hirigoyen MF. Le harcèlement morale. París: Syros; 1994.

5. Einarsen S, Hoel H, Zapf D, Cooper CL, editors. Bullying and emotional abuse in the workplace: international perspectives in research and practice. London: Taylor \& Francis; 2003.

6. Lorenz K. Hier bin ich-wo bist Du? Ethologie der grauganz. München: Piper; 1991.

7. Cardoso de Oliveira LR. Direitos, insulto e cidadania (Existe violência sem agressão moral). Série Antropologia. Departamento de Antropologia. Universidade de Brasília; 2005.

8. Girard R. El chivo expiatorio. Barcelona: Anagrama; 2001.

9. Hoel H, Einarsen S. Shortcomings of antibullying regulations: the case of Sweden. Eur J Work Org Psych. 2010;19(1):30-50.

10. Di Martino V, Hoel H, Cooper CL. Preventing violence and harassment in the workplace (European Foundation for the Improvement of Living and Working Conditions). Luxemburg: Office for Official Publications of the European Communities; 2003.

11. Harvey S, Keashly L. Emotional abuse: how the concept sheds light on the understanding of psychological harassment (in Québec). Pistes. 2005;7(3).

12. Barren O. Preventing bullying at work. The development of a proactive enforcement approach by an Australian Occupational Health and Safety regulator. Proceedings from the 5th International Conference on Bullying and Harassment in the Workplace. Dublin: Trinity College; 2006.

13. Gimeno Lahoz R. La presión laboral tendenciosa. El mobbing desde la óptica de un juez. Valladolid: Lex Nova; 2004.

14. Soetzer U, Ahlberg G, Bergman P, Hallsten L, Lundberg I. Working conditions predicting interpersonal relationship problem at work. Eur J Work Org Psych. 2009;18(4):424-41.

15. Leymann H. Mobbing - Psychological violence at work places. Lund: Studentlitteratur; 1986.

16. Leymann H. Mobbing. La persécution au travail. Paris: Seuil; 1996.

17. Leymann H, Gustafsson A. Mobbing at work and the development of Post-traumatic Stress Disorders. Eur J Work Org Psych. 1996;5:251-77.

18. Mikkelsen EG, Einarsen S. Relationships between exposure to bullying at work and psychological and psychosomatic health complaints: the role of state negative affectivity and generalized self-efficacy. Scand J Psychol. 2002;43(5):397-405.

19. Fidalgo A, Piñuel I. La escala Cisneros como herramienta de valoración del mobbing. Psicothema. 2004;16(4):615-24.

20. Topa G, Depolo M, Morales JF. Acoso laboral: meta-análisis y modelo integrador de sus antecedentes y consecuencias. Psicothema. 2007;19(1):88-94.

21. Magalhães Guimarães LA, Rimoli A. "Mobbing" (assédio psicológico) no trabalho: uma síndrome psicossocial multidimensional. Psicologia: teoria e pesquisa. 2006;22(2):183-92.

22. González-de-Rivera JL, Rodríguez-Abuín MJ. Cuestionario de estrategias de acoso psicológico: el LIPT-60 (Leymann Inventory of Psychological Terrorization) en versión española. Psiquis. 2003;24(2):59-69.

23. González-de-Rivera JL. El maltrato psicológico. Como defenderse del bullying, el mobbing y otras formas de acoso. Madrid: Espasa-Calpe; 2003.

24. González-de-Rivera JL. Claves del mobbing. Madrid: EOS; 2005.

25. González-de-Rivera JL, Rodríguez-Abuín MJ. LIPT-60. Cuestionario de Estrategias de Acoso en Trabajo. Madrid: EOS; 2005.

26. González de Rivera JL, Rodríguez-Abuín M. Acoso psicológico en el trabajo y psicopatología: un estudio con el LIPT-60 y el SCL 90-R. Rev Psicol Trab Organ. 2006;22(3):397-412.

27. Einarsen S, Raknes BI. Harassment in the workplace and the victimization of men. Violence Vict. 1997;12:247-63.

28. Piñuel I, Oñate A. La incidencia del mobbing o acoso psicológico en el trabajo en España. Lan Harremanak. 2002;7(II):35-62.

29. Piñuel I. Informe Cisneros V. La incidencia del mobbing o acoso psicológico en el trabajo en la administración (AEAT e IGAE). Disponível em: http://www.acosomoral.org/pdf/informeCisnerosV.pdf.

30. Puckett MB, Aikins JW, Cillessen AH. Moderators of the association between relational aggression and perceived popularity. Aggress Behav. 2008;34(6):563-76.

31. Green VA, Cillessen AH, Rechis R, Patterson MM, Hughes JM. Social problem solving and strategy use in young children. J Genet Psychol. 2008;169(1):92-112.

32. Kleinman A, Hall-Clifford R. Stigma: a social, cultural and moral process. J Epidemiol Community Health. 2009;63(6):418-9.

33. Yang LH, Kleinman A. "Face" and the embodiment of stigma in China: the cases of schizophrenia and AIDS. Soc Sci Med. 2008;67(3):398-408.

34. Yang LH, Kleinman A, Link BG, Phelan JC, Lee S, Good B. Culture and stigma: adding moral experience to stigma theory. Soc Sci Med. 2007;64(7):1524-35.

35. Patel V, Saraceno B, Kleinman A. Beyond evidence: the moral case for international mental health. Am J Psychiatry. 2006;163(8):1312-5.

36. González de Rivera JL, López García-Silva JA. La valoración médicolegal del mobbing o acoso laboral. Psiquis. 2003;24:107-14.

37. Agervold M. Bullying at work: a discussion of definitions and prevalence, based on an empirical study. Scand J Psychol. 2007;48(2):161-72.

38. Moreno Jiménez B, Rodríguez Muñioz A, Martínez Gamarra M, Gálvez Herrer M. Assessing workplace bullying: Spanish validation of a reduced version of the Negative Acts Questionnaire. Span J Psychol. 2007;10(2):449-57.

39. Mikkelsen EG, Einarsen S. Bullying in Danish worklife: prevalence and health correlates. Eur J Work Org Psych. 2001;10:393-413.

40. Zapf D, Einarsen S, Hoel H, Vartia M. Empirical findings on bullying in the workplace. In: Einarsen S, Hoel H, Zapf D, Cooper CL, editors. Bullying and emotional abuse in the workplace. International perspectives in research and practice. London/New York: Taylor and Francis; 2003, p. 222-41. 
41. Di Martino V. Work-related violence. New York: Westdeutscher Verlag Wesbaden and Westview Press; 2002.

42. Elovainio Marko, Kivimäki Mika, Vahtera Jussi. Organizational justice: evidence of a new psychosocial predictor of health. Am J Public Health. 2002;92(1):105-8.

43. Kivimäki M, Elovainio M, Vahtera J. Workplace bullying and sickness absence in hospital staff. Occup Environ Med. 2000;57(10):656-60.

44. Voss M, Floderus B, Diderichsen F. Physical, psychosocial, and organisational factors relative to sickness absence: a study based on Sweden Post. Occup Environ Med. 2001;58(3):178-84.

45. Vartia MA. Consequences of workplace bullying with respect to the wellbeing of its targets and the observers of bullying. Scand J Work Environ Health. 2001;27(1):63-9.

46. Hemingway H, Marmot M. Evidence based cardiology: psychosocial factors in the aetiology and prognosis of coronary heart disease. Systematic review of prospective cohort studies. BMJ. 1999;318(7196):1460-7.

47. Amick BC 3rd, Kawachi I, Coakley EH, Lerner D, Levine S, Colditz GA. Relationship of job strain and iso-strain to health status in a cohort of women in the United States. Scand J Work Environ Health. 1998;24(1):54-61.

48. Weinberg A, Creed F. Stress and psychiatric disorder in healthcare professionals and hospital staff. Lancet. 2000;355(9203):533-7.

49. Koskenvuo M, Kaprio J, Rose RJ, Kesäniemi A, Sarna S, Heikkilä K, Langinvainio $\mathrm{H}$. Hostility as a risk factor for mortality and ischemic heart disease in men. Psychosom Med. 1988;50(4):330-40.

50. Scott MJ, Stradling SG. PTSD without the trauma. Br J Clin Psychol. 1994;33:71-4

51. Kreiner B, Sulyok C, Rothenhäusler HB. Does mobbing cause posttraumatic stress disorder? Impact of coping and personality. Neuropsychiatr. 2008;22(2):112-23.

52. Bonafons C, Jehel L, Coroller-Béquet A. Specificity of the links between workplace harassment and PTSD: primary results using court decisions, a pilot study in France. Int Arch Occup Environ Health. 2009;82(5):663-8.

53. Gilioli R, Campanini P, Fichera GP, Punzi S, Cassitto MG. Emerging aspects of psychosocial risks: violence and harassment at work. Med Lav. 2006;97(2):160-4.

54. Hansen AM, Hogh A, Persson R, Karlson B, Garde AH, Ørbaek P. Bullying at work, health outcomes, and physiological stress response. J Psychosom Res. 2006;60(1):63-72.

55. Pompili M, Lester D, Innamorati M, De Pisa E, Iliceto P, Puccinno M, et al. Suicide risk and exposure to mobbing. Work. 2008;31(2):237-43.

56. Kivimäki M, Virtanen M, Vartia M, Elovainio M, Vahtera J, KeltikangasJärvinen L. Workplace bullying and the risk of cardiovascular disease and depression. Occup Environ Med. 2003;60(10):779-83.

57. Lindeberg SI, Eek F, Lindbladh E, Östergren PO, Hansen AM, Karlson BB. Exhaustion measured by the SF- 36 vitality scale is associated with a flattened diurnal cortisol profile. Psychoneuroendocrinology. 2008;33:471-7.
58. Niedhammer I, David S, Degioanni S. Association between workplace bullying and depressive symptoms in the French working population. J Psychosom Res. 2006;61(2):251-9.

59. Jarreta BM, García-Campayo J, Gascón S, Bolea M. Medico-legal implications of mobbing. A false accusation of psychological harassment at the workplace. Forensic Sci Int. 2004;146(Suppl):S17-8.

60. American Psychiatric Association. Diagnostical and Statistical Manual of Mental Disorders. DSM-IV-TR. Barcelona: Massón; 2000.

61. Glasø L, Matthiesen SB, Nielsen MB, Einarsen S. Do targets of workplace bullying portray a general victim personality profile? Scand J Psychol. 2007;48(4):313-9.

62. Balducci C, Alfano V, Fraccaroli F. Relationships between mobbing at work and MMPI-2 personality profile, posttraumatic stress symptoms, and suicidal ideation and behavior. Violence Vict. 2009;24(1):52-67.

63. Brousse G, Fontana L, Ouchchane L, Boisson C, Gerbaud L, Bourguet D, et al. Psychopathological features of a patient population of targets of workplace bullying. Occup Med (Lond). 2008;58(2):122-8.

64. Moreno-Jiménez B, Rodríguez-Muñoz A, Morante ME, Garrosa E, Rodríguez-Carvajal R, et al. Evaluación del acoso psicológico en el trabajo: desarrollo y estudio exploratorio de una escala de medida. Univ Pscyhol. 2008;7(2):335-45.

65. Trijueque DG, Delgado Marina S. Acoso laboral y trastornos de la personalidad: un estudio con el MCMI-II. Clínica y Salud. 2008;19(2):191-204.

66. Mikkelsen EG, Einarsen S. Bullying in Danish work-life: prevalence and health correlates. Eur J Work Organ Psychol. 2001;10(4):393-413.

67. Mikkelsen EG, Einarsen S. Basic assumptions and symptoms of posttraumatic stress among victims of bullying at work. Eur J Work Organ Psychol. 2002;11(1):87-111.

68. Rodríguez-Abuín MJ, González de Rivera JL. Diagnóstico diferencial del paranoidismo en las víctimas de acoso. Interpsiquis. 2006. Disponível em: http://www.psiquiatria.com/articulos/estres/25255/ [accessed July 13 2009].

69. Nielsen MB, Matthiesen SB, Einarsen S. Sense of coherence as a protective mechanism among targets of workplace bullying. J Occup Health Psychol. 2008;13(2):128-36.

70. Martínez-Hernáez A. What's behind the symptom? On psychiatric observation and anthropological understanding. London \& New York: Routledge; 2000.

71. Kleinman A. Patients and healers in the context of culture. Berkeley: University of California Press; 1980.

72. Good B. Medicina, racionalidad y experiencia. Una perspectiva antropológica. Barcelona: Bellaterra Ediciones; 2000.

73. Menezes Júnior A, Moreira-Almeida A. O diagnóstico diferencial entre experiências espirituais e transtornos mentais de conteúdo religioso. Rev Psiq Clín. 2009;36(2):75-82.

74. Almeida AM, Neto FL. Diretrizes metodológicas para investigar estados alterados de consciência e experiências anômalas. Rev Psiq Clín. 2003;30(1):21-8. 